\title{
Les jeunes aiment \\ - encore - \\ la recherche
}

Jean-Claude Weill

L'Académie des sciences organise chaque année sous l'égide de la section «Sciences chimiques, biologiques et médicales » un concours appelé «Les grandes avancées françaises en biologie présentées par leurs auteurs » pour lequel peuvent postuler tous les jeunes chercheurs travaillant en France, ayant publié dans l'année un article dans un des grands journaux scientifiques internationaux tels que Science ou Nature.

Le jury présidé par notre secrétaire perpétuelle Pascale Cossart décerne cinq prix et reçoit en moyenne 70 à 80 articles, les candidats devant être les premiers auteurs du travail et donc ceux qui ont en accompli la plus grande part. Ces jeunes chercheurs ont en moyenne de 30 à 35 ans et la plupart d'entre eux sont rémunérés par une bourse en attendant d'obtenir éventuellement un poste à l'Inserm ou au CNRS. Ce poste leur assurera un salaire qui sera initialement d'environ un peu plus de 2000 euros par mois. Voilà donc, chaque année en France, des jeunes passionnés par la recherche, «sur-diplomés » et «sur-formés 》 - car ils ont tous au minimum un doctorat en sciences et plusieurs années de stage post-doctoral - qui, avec un engagement total, arrivent à publier leurs travaux dans les plus grandes revues scientifiques du monde.

Que dire devant ces parcours remarquables? Qu'il s'agit de moines qui ont choisi d'entrer dans les ordres? Non, ce sont simplement des jeunes habités par la science et totalement impliqués dans cette aventure qu'est la recherche scientifique.

Notre pays garde cette tradition du savoir et du goût du risque, cette passion de la jeunesse, qui permet d'ouvrir de nouveaux champs de la connaissance.

On nous propose une start-up nation? Pourquoi pas, après tant d'essais pour changer notre pays. Mais une start-up, c'est d'abord une nouvelle idée et un/de nouveau $(x)$ brevet(s) et donc, en amont, une recherche fondamentale et parfois appliquée, menée dans des laboratoires la plupart du temps académiques.

C'est une banalité de le dire, mais la recherche est l'avenir d'un pays et il suffit pour le comprendre de voir l'investissement que fait la Chine pour faire revenir ses meilleurs chercheurs qui travaillent à l'étranger.

Vignette (Photo @ Inserm-Patrick Delapierre).

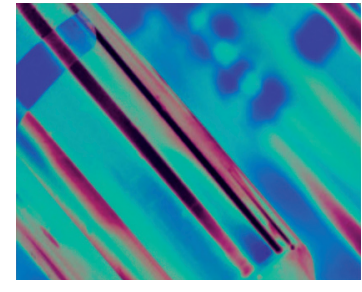

Professeur d'Immunologie, membre de l'Académie des Sciences, INEM-Institut NeckerEnfants Malades, Inserm U1151/ CNRS UMR8253, Faculté de médecine Paris-Descartes, Site Broussais, 14, rue Maria Helena Vieira Da Silva, 75993 Paris, France.

jean-claude.weill@inserm.fr

Mais que donc proposer après ces belles paroles?

- Mettre le budget de la recherche au niveau de celui des autres pays développés, soit autour de $3 \%$ du produit intérieur brut (PIB).

- Revaloriser les salaires et les aligner sur ceux de nos concurrents et néanmoins collègues à l'étranger.

- Supprimer de mauvaises règles qui peuvent se justifier mais dont l'effet s'avère dramatique dans le monde de la recherche académique : I'obligation de réaliser sa thèse en 3 ans, qui oblige à des projets faisables sans prise de risque et fait de nos thésards des candidats qui ont de moins en moins accès aux grands laboratoires pour leur stage post-doctoral. Adapter la loi Sauvadet à notre profession, écrite afin de limiter à 6 ans le recours à des contrats à durée déterminée (CDD) pour remplir des fonctions pérennes dans la fonction publique. Cette loi est contre-productive dans le domaine de la recherche et est en train de décapiter nos équipes en jeunes chercheurs et en IT (ingénieurs et techniciens). En effet, pour être un candidat crédible à un organisme de recherche, dont l'âge moyen de recrutement est de 35 ans, il faut, après sa thèse en sciences, faire un ou deux stages postdoctoraux, puis ensuite passer quelques années dans un laboratoire, soit un parcours pratiquement impossible à accomplir en 6 ans. La loi Sauvadet oblige donc ces candidats à terminer celui-ci à l'étranger, avec le risque pour les meilleurs qu'ils ne reviennent jamais travailler en France. Elle oblige également les laboratoires à se défaire de jeunes IT bien formés, compétents, sans que des fonctions pérennes puissent leur être proposées.

Il est grand temps de passer aux actes ! $\diamond$

Young people like - still - research

\section{LIENS D'INTÉRÊT}

L'auteur déclare n'avoir aucun lien d'intérêt concernant les données publiées dans cet article. 